Hans Grimmer* and Bernard Delley

\title{
Density functional theory calculations of merohedric twinning in $\mathrm{KLiSO}_{4}$
}

https://doi.org/10.1515/zkri-2018-2126

Received September 3, 2018; accepted October 22, 2018; published online December 8, 2018

\begin{abstract}
Density functional theory (DFT) calculations have been performed on five models of periodic, polysynthetic twin interfaces in the ambient-temperature phase of $\mathrm{KLiSO}_{4}$, which has space group $\mathrm{PG}_{3}$. The models represent the three merohedric twin laws $(m \| z, 2 \perp z$ and $\overline{1})$ with boundary plane (1 $0 \overline{1} 0)$, also with boundary plane ( $\left.\begin{array}{llll}0 & 0 & 0 & 1\end{array}\right)$ in case of $m$, and with boundary plane $\left(\begin{array}{lll}1 & \overline{2} & 1\end{array}\right)$ in case of $\overline{1}$. The models satisfy stoichiometry at the boundary plane and maintain the fourfold coordination of the Li and $S$ atoms and the twofold coordination of the oxygen atoms. Relaxed lattice parameters and atomic positions were determined by DFT, using the $\mathrm{DMol}^{3}$ code with functional PBEsol. The energy difference between polysynthetic twin and single crystal per primitive cell of the twin is $0.0009 \mathrm{eV}$ for $m(0001), 0.09 \mathrm{eV}$ for $\overline{1}(10 \overline{1} 0), 0.58 \mathrm{eV}$ for $m(10 \overline{1} 0)$ and $0.55 \mathrm{eV}$ for $2(10 \overline{1} 0)$. In $\mathrm{KLiSO}_{4}$ crystals grown from aqueous solutions the first twin was frequently observed, similarly also the second twin in Cr-doped crystals, whereas the third twin appeared only rarely and the fourth was not observed. Not only for $\mathrm{KLiSO}_{4}$ but also for quartz, the energy of twins and the frequency of their occurrence are closely connected for crystals grown from aqueous solutions, whereas for the formation of transformation twins the availability of twin nuclei plays a major role.
\end{abstract}

Keywords: density functional theory; growth twin; potassium lithium sulfate; quartz; twinning.

Dedicated to: Prof. emerit. Theo Hahn, RWTH Aachen University, Germany († 12 Feb. 2016).

*Corresponding author: Hans Grimmer, Multiscale Materials Experiments, Research with Neutrons and Muons, Paul Scherrer Institut, Forschungsstrasse 111, CH-5232 Villigen PSI, Switzerland, E-mail: hans.grimmer@psi.ch

Bernard Delley: Scientific Computing and Modelling, Research with Neutrons and Muons, Paul Scherrer Institut, Forschungsstrasse 111, $\mathrm{CH}-5232$ Villigen PSI, Switzerland

\section{Introduction}

A long-time research project of the late Theo Hahn and his collaborators at the RWTH Aachen concerned $\mathrm{KLiSO}_{4}$, its twin domains, twin boundaries and phase transitions [1-3].

Crystals of $\mathrm{KLiSO}_{4}$, space group $\mathrm{P}_{3}$ at room temperature, can exhibit 'twinning by merohedry' with the three twin laws

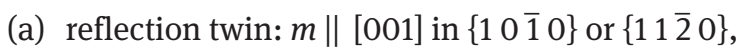

(b) rotation twin: $2 \|\langle 100\rangle$ or $\langle 210\rangle$,

(c) inversion twin: $\overline{1}$ or $m \perp[001]$.

The polarity (sign of the pyro- and piezoelectric effect) of the two individuals of a twin is the same for twin (a) and reversed for (b) and (c); the chirality (sense of optical rotation) is the same for twin (b) and reversed for (a) and (c) (cf. table 1 of [1]).

Atomic structure models of twins (a), (b), (c) with

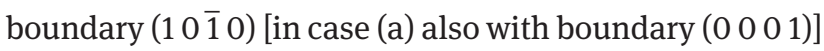
were proposed in [1] and further illustrated in [4].

In the following we denote a twin boundary (hkil) associated with twin laws (a), (b) and (c) by m(hkil), 2(hkil) and $\overline{1}(h k i l)$, respectively.

$\mathrm{KLiSO}_{4}$ crystals were grown by evaporation of aqueous solutions of $\mathrm{K}_{2} \mathrm{SO}_{4}$ and $\mathrm{Li}_{2} \mathrm{SO}_{4} \times \mathrm{H}_{2} \mathrm{O}$. The optically best crystals were obtained replacing $\mathrm{K}_{2} \mathrm{SO}_{4}$ partly by $\mathrm{K}_{2} \mathrm{CrO}_{4}$; only about $0.5 \%$ of $\mathrm{S}$ was replaced by $\mathrm{Cr}$ in these crystals [1-4]. Cr doping affected the color and morphology of the crystals but not the phase transformations; it affected the occurrence of inversion twins. Growing crystals up to $3 \mathrm{~cm}$ size and sufficiently perfect for X-ray topography took about 8 weeks.

Reflection twins with boundary ( $\left.\begin{array}{llll}0 & 0 & 0 & 1\end{array}\right)$ were frequently found, sometimes with boundaries traversing the whole crystal and sometimes in polysynthetic form with lamellae often too thin to be resolved by X-ray topography. Reflection twins with boundary (10 $\overline{1} 0)$ were only observed as lateral bound of ( $\left.\begin{array}{llll}0 & 0 & 0 & 1\end{array}\right)$ lamellae. Inversion twins with extended ( $10 \overline{1} 0$ ) boundaries were found in Cr-doped crystals (see fig. 3 in [3]). Rotation twins were not found.

The polarization optic photographs given in fig. 5.1.10 of [4] show numerous lines approximately lying in the three $\{11 \overline{2} 0\}$ planes. They were interpreted as boundaries 
of inversion twins. The photographs were published also as fig. $5 \mathrm{c}, \mathrm{d}$ of [3], where it is stated that the lines also might represent growth dislocations. The plane $\left(\begin{array}{llll}1 & 1 & \overline{2} & 0\end{array}\right)$ can be approximated by staggering: $\left(\begin{array}{llll}1 & 1 & \overline{2} & 0\end{array}\right)=\left(\begin{array}{llll}1 & 0 & \overline{1} & 0\end{array}\right)+$ (0 $1 \overline{1} 0)$. The lines on the photographs do not suggest staggered planes, so that we developed a structure model for an inversion twin with boundary $(11 \overline{2} 0)$.

\section{DFT calculations of $\mathrm{KLiSO}_{4}$ crystals}

The room temperature phase of $\mathrm{KLiSO}_{4}$ (also known as phase III) has space group $\mathrm{PG}_{3}$ (\#173). Its structure at $293 \mathrm{~K}$ according to [5] is shown in Figure 1.

The hexagonal lattice can be characterized by the lattice parameters $c$ and $a$ or, alternatively, by $c / a$ and
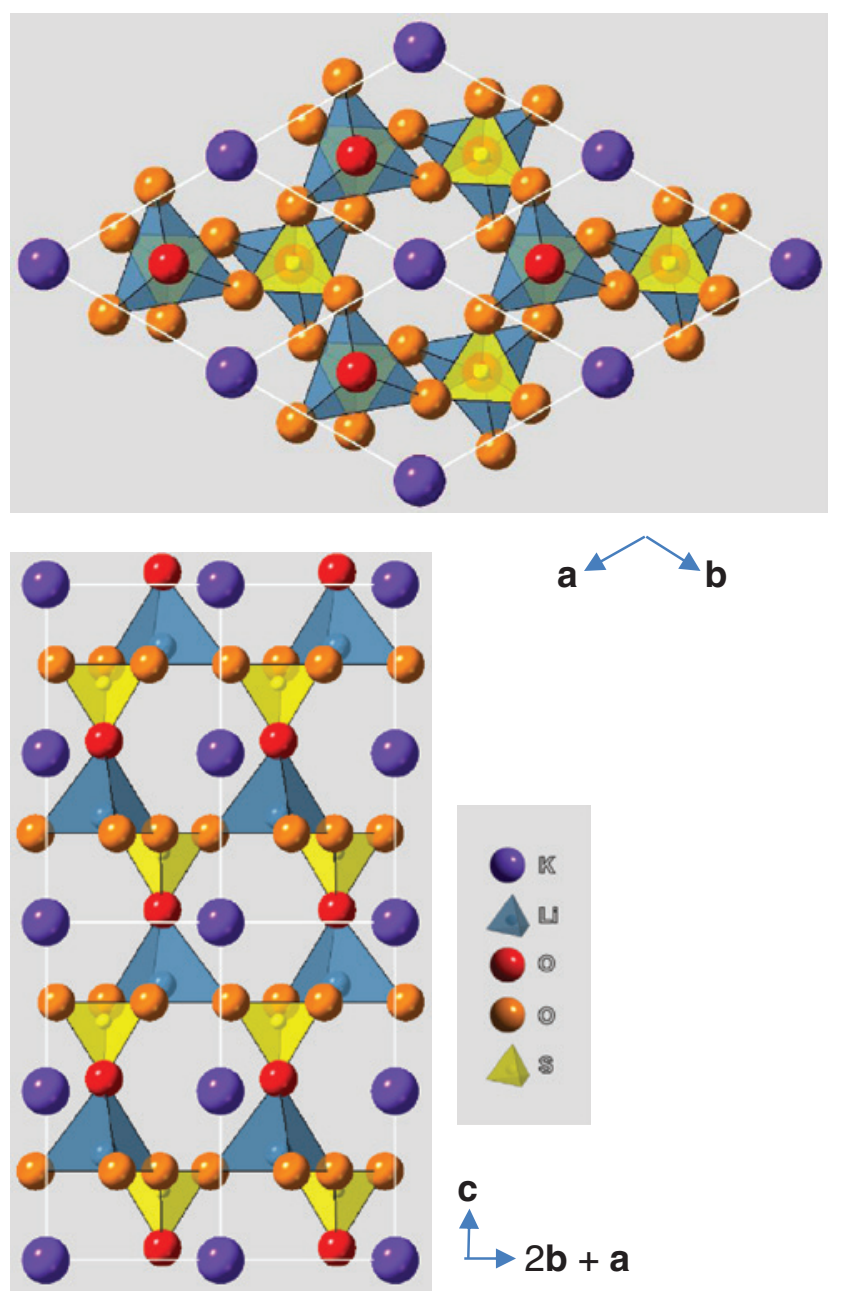

Fig. 1: Structure of $\mathrm{KLiSO}_{4}$ at room temperature projected along c (top) and along a (bottom). The yellow oxygen tetrahedra contain $S$ inside, the blue-green ones Li. Each tetrahedron is connected at its four vertices with tetrahedra of the other color. $\mathrm{K}$ is at Wyckoff position $2 \mathrm{a}, \mathrm{S}, \mathrm{Li}$ and red $\mathrm{O}$ at $2 \mathrm{~b}$, orange $\mathrm{O}$ at $6 \mathrm{c}$. the volume $V=1 / 2 \sqrt{ } 3 a^{2} c$ of the primitive cell. The elastic stiffness matrix is symmetric and determined by five constants $C_{11}=C_{22}, C_{12}, C_{13}=C_{23}, C_{33}$ and $C_{44}=C_{55^{\circ}} C_{66}$ is related to these by $C_{66}=1 / 2\left(C_{11}-C_{12}\right)[6]$.

Our DFT calculations used the $\mathrm{DMol}^{3}$ code developed by Delley [7]. As a first step, several functionals were tested to find out which one best reproduced the experimental values $c / a, V$ and the $C_{i j}$ of phase III. The calculations used as input ICSD-file 36470.cif, based on the $\mathrm{KLiSO}_{4}$ structure at $293 \mathrm{~K}$, as determined in [5] (see Table 1). In all cases a $4 \times 4 \times 4 \Gamma$-centered mesh in $\mathrm{k}$-space was used as well as the default local orbital basis set DNP [7] with cutoff radii for the basic functions equal to $13.38 a_{0}$ for $\mathrm{K}, 11.68 a_{0}$ for $\mathrm{Li}$, to $7.32 a_{0}$ for $\mathrm{S}$ and $8.58 a_{0}$ for $\mathrm{O}, a_{0}$ denoting the Bohr radius.

The earliest developed and simplest functional tested was the local-density-functional approximation (LDA) of the Perdew Wang Correlation (PWC) [9] plus local exchange. It produced the least good approximation to the experimental values of $C_{i j}$ and $c / a$. The generalized gradient approximation (GGA) functionals PBE [10] and PBEsol [11] gave the best approximations to $C_{i j}$ and $c / a$; the newest and most sophisticated meta-GGA functional SCAN [12] lying in between.

For the subsequent twin calculations PBEsol was selected, which had led to values of $c / a$ and $V$ closer to the experimental ones than PBE. In the framework of the Materials Project [13] another DFT calculation of phase III has been made using the PBE functional with the VASP

Tab. 1: Fractional coordinates of the atoms of $\mathrm{KLiSO}_{4}$ at $293 \mathrm{~K}$ according to [5].

\begin{tabular}{|c|c|c|c|}
\hline & $\mathbf{x}$ & $y$ & z \\
\hline \multicolumn{4}{|l|}{$2 a$} \\
\hline K1 & 0 & 0 & 0 \\
\hline K2 & 0 & 0 & 0.5 \\
\hline \multicolumn{4}{|l|}{$2 b$} \\
\hline Li1 & $1 / 3$ & $2 / 3$ & 0.8150 \\
\hline Li2 & $2 / 3$ & $1 / 3$ & 0.3150 \\
\hline \multicolumn{4}{|l|}{$2 b$} \\
\hline S1 & $1 / 3$ & $2 / 3$ & 0.2063 \\
\hline S2 & $2 / 3$ & $1 / 3$ & 0.7063 \\
\hline \multicolumn{4}{|l|}{$2 b$} \\
\hline 01 & $1 / 3$ & $2 / 3$ & 0.0367 \\
\hline 02 & $2 / 3$ & $1 / 3$ & 0.5367 \\
\hline \multicolumn{4}{|l|}{$6 c$} \\
\hline 03 & 0.0601 & 0.4075 & 0.2634 \\
\hline 04 & 0.5925 & 0.6526 & 0.2634 \\
\hline 05 & 0.3474 & 0.9399 & 0.2634 \\
\hline 06 & 0.9399 & 0.5925 & 0.7634 \\
\hline 07 & 0.4075 & 0.3474 & 0.7634 \\
\hline 08 & 0.6526 & 0.0601 & 0.7634 \\
\hline
\end{tabular}

The first column gives the Wyckoff letter of space group $P 6_{3}$ (\#173) [8]. 
Tab. 2: Experimental and DFT results for the lattice parameters and the elastic stiffness matrix of $\mathrm{KLiSO}_{4}$ phase III.

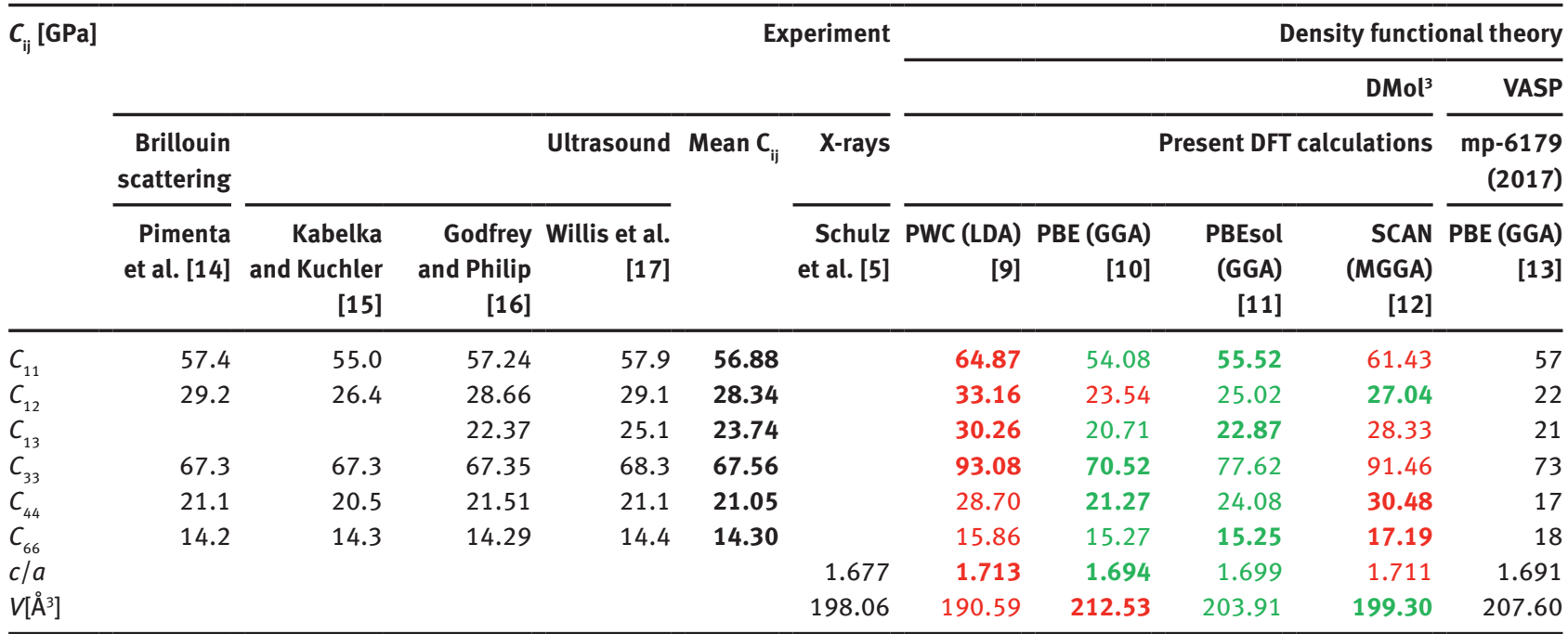

The $\mathrm{DMol}^{3}$ results have been marked bold-green, green, black, red and bold-red with increasing deviation from the (mean) experimental value.

code instead of $\mathrm{DMol}^{3}$. Table 2 shows that the two codes led to similar results.

\section{DFT calculations of twins in $\mathrm{KLiSO}_{4}$ crystals}

\section{Polysynthetic twin models and illustration of DFT results}

The DFT calculations of $\mathrm{KLiSO}_{4}$ made use of periodic boundary conditions: a primitive cell of the $\mathrm{KLiSO}_{4}$ crystal contains two formula units, i.e. 14 atoms. In order to deal with a finite number of atoms also in case of twins, sequences of periodic polysynthetic twins with increasing lamellae thickness were considered, containing $28 N$ atoms, $N=1,2,3$, 4. A primitive cell of the polysynthetic twin contains $14 \mathrm{~N}$ atoms of each of the two individuals.
$\mathrm{DMol}^{3}$ with functional PBEsol was used in the first step of our calculations to obtain the model for bulk $\mathrm{KLiSO}_{4}$ described in Table 2. This model was used to construct the polysynthetic twin models that served as input to the second step, in which the twin models were relaxed.

\section{Reflection twin with boundary $\left(\begin{array}{llll}0 & 0 & 0 & 1\end{array}\right): m\left(\begin{array}{llll}0 & 0 & 0 & 1\end{array}\right)$}

The structure model illustrated in fig. 5.5 .1 of [4] has a boundary plane ( $\left.\begin{array}{llll}0 & 0 & 0 & 1\end{array}\right)$. The boundary contains only $\mathrm{K}$ atoms. (See also fig. 11a of [1] and fig. 3.3.10.8 of [18].)

The polysynthetic twin models have a hexagonal lattice with basis vectors $\mathbf{a}, \mathbf{b}$ and $\mathbf{C}=2 N \mathbf{c}$. The twin models have space group P31c (\#159). The two individuals of the twin are related by the $c$-glide plane. For the boundary $\left(\begin{array}{llll}0 & 0 & 0 & 1\end{array}\right)$ the reflection twin model differs from a single crystal only by the position of the $\mathrm{O}$ atoms in Wyckoff position $6 \mathrm{c}$, the positions of $\mathrm{K}, \mathrm{Li}, \mathrm{S}$ and of $\mathrm{O}$ in Wyckoff position $2 \mathrm{~b}$ being unaffected by twinning. Figure 2 shows the DFT result for $N=2$.

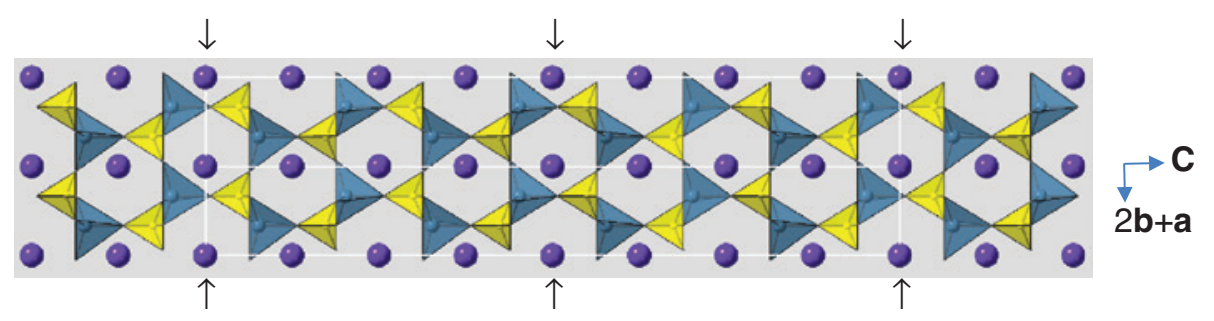

Fig. 2: DFT result for the reflection twin with boundary $\left(\begin{array}{llll}0 & 0 & 0 & 1\end{array}\right)$ projected $\|$ a. The yellow oxygen tetrahedra contain $\mathrm{S}$ inside, the blue-green ones $\mathrm{Li}$. The arrows indicate the boundaries; they are perpendicular to the plane of the drawing. (The difference between input model and DFT result is too small to be visible.) 


\section{Twins with boundary $\left(\begin{array}{lll}1 & 0 & 1\end{array}\right)$}

The structure models illustrated in figs. 5.5.2-5.5.4 of [4]

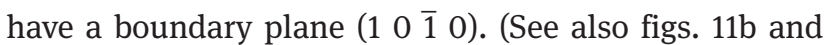
12a, b of [1].) The boundary contains only $\mathrm{K}$ atoms. The $\mathrm{K}$ atoms in these twin models are at the same positions as in a single crystal.

The polysynthetic twin models with this boundary have an orthorhombic lattice with basis vectors $\mathbf{A}=N(2 \mathbf{a}+\mathbf{b}), \mathbf{b}$ and $\mathbf{c}$.

\section{Reflection twin: $m(10 \overline{1} 0)$}

Structure models of this twin are illustrated in fig. 5.5.2 of [4] and in fig. 11b of [1]. The polysynthetic twin models have space group Pca2 ${ }_{1}$ (\#29). The two individuals of the twin are related by the $c$-glide plane. Figure 3 shows the DFT result for $N=4$.

\section{Rotation twin: $2\left(\begin{array}{lll}1 & 0 & 1\end{array}\right)$}

Structure models of this twin are illustrated in fig. 5.5.3 of [4] and in fig. 12b of [1]. The polysynthetic twin models have space group $P 22_{1} 22_{1}(\# 18)$. The two individuals of the twin are related by the $180^{\circ}$ rotation $\|$ b. Figure 4 shows the DFT result for $N=4$.

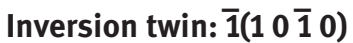

Structure models of this twin are illustrated in fig. 5.5.4 of [4] and in fig. 12a of [1]. The polysynthetic twin models have space group \#14 with cell choice $1\left(P 112_{1} / a\right)$ if $N$ is even, cell choice $2\left(P 112_{1} / n\right)$ if $N$ is odd [8]. The two individuals of the twin are related by inversions at points $1 / 2(u, v, w)$, where $u, v, w$ are integers. Figure 5 shows the DFT result for $N=4$.

\section{Inversion twin with boundary (1 $1 \overline{2} 0): \overline{1}(11 \overline{2} 0)$}

(1 $11 \overline{2}$ 0) planes containing $\mathrm{K}$ also contain $\mathrm{Li}, \mathrm{S}$ and $\mathrm{O}$ in Wyckoff position $2 \mathrm{~b}$. In our starting model of the polysynthetic twin we placed these atoms alternately in the positions for one or the other of the two individuals. These models have space group \#14 with cell choice $1\left(P 112_{1} / a\right)$ if $N$ is even, cell choice $2\left(P 112_{1} / n\right)$ if $N$ is odd [8].

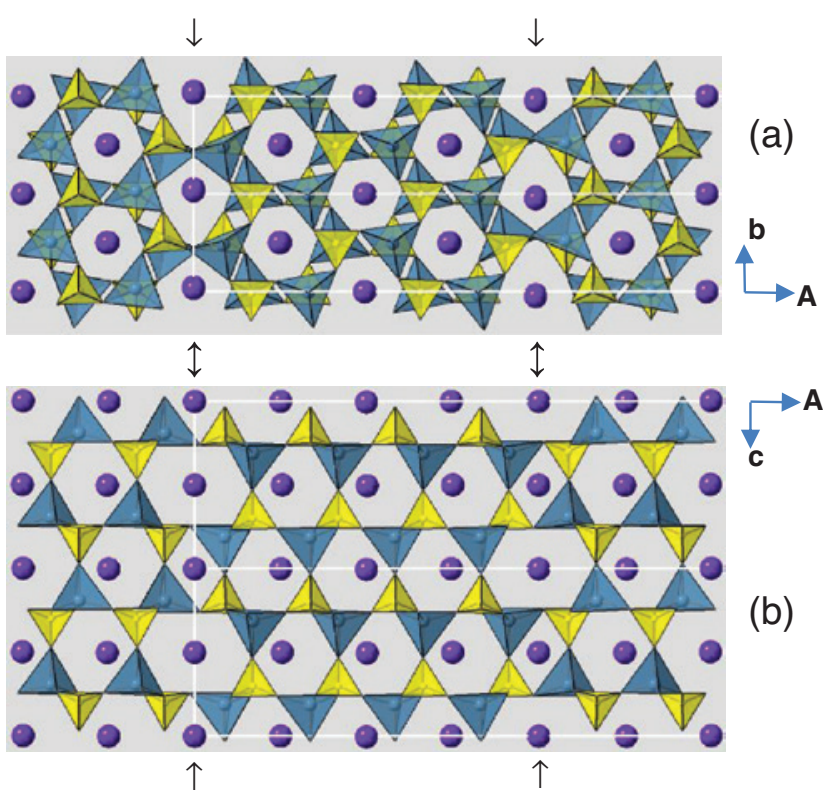

Fig. 4: DFT results for the rotation twin with boundary (1 $0 \overline{1} 0)$ projected || c (a) and || b (b). The arrows and double arrows indicate the boundaries; they are perpendicular to the planes of the drawings. The width of a primitive cell (marked white) is twice the distance between neighboring boundaries. In the unrelaxed structure projected || c (shown in fig. 5.5.3 (a) of [4]) the blue-green triangles that do not cross a boundary were equilateral, those that cross a boundary were far from equilateral. In the relaxed structure shown in (a) the differences between triangles are smaller. Consequently, only the gray hexagon at the center of the figure remains nearly regular. 


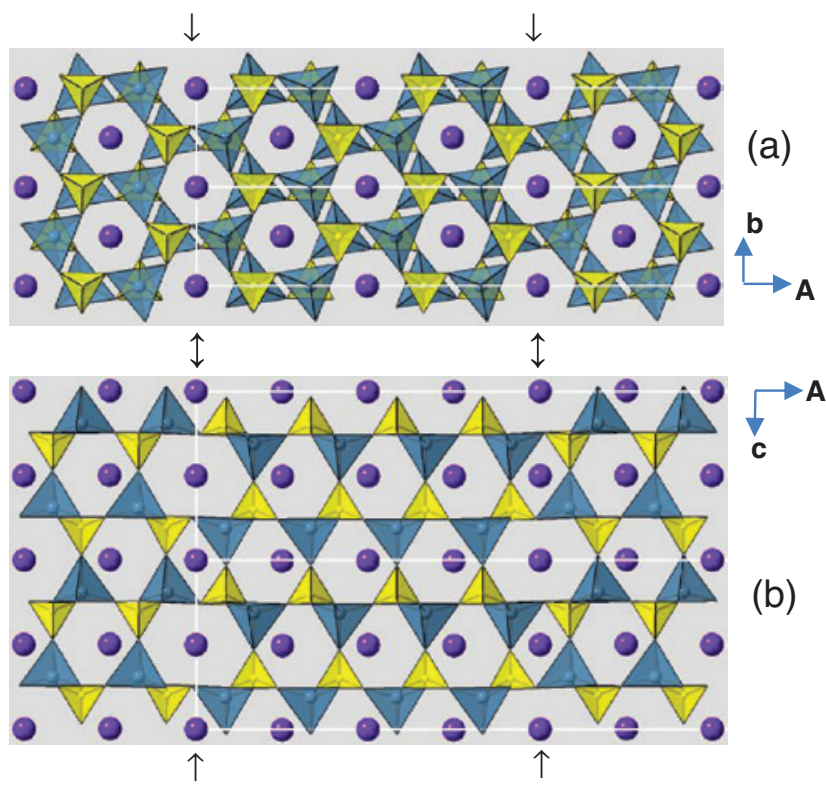

Fig. 5: DFT results for the inversion twin with boundary (1 $01 \overline{1} 0)$ projected || c (a) and || b (b). The arrows and double arrows indicate the boundaries; they are perpendicular to the planes of the drawings. The width of a primitive cell (marked white) is twice the distance between neighboring boundaries.

The polysynthetic twin models with this boundary have a pseudo-orthorhombic lattice with basis vectors $\mathbf{A}^{\prime}=N(\mathbf{a}+\mathbf{b}), \mathbf{B}^{\prime}=\mathbf{b}-\mathbf{a}, \mathbf{c}$. Figure 6 shows the DFT result for $N=4$.

\section{Summary of DFT results}

In the DFT calculations the lattice parameters in the boundary plane were kept fixed because they will approach bulk values as $N$ increases. To minimize energy, the atom positions and the remaining lattice parameters are varied within the restrictions imposed by the symmetry of the polysynthetic twin. This means for the lattice that a stretch perpendicular to the boundary plane is allowed and, in case of monoclinic symmetry, a shear parallel to the boundary plane and normal to the monoclinic axis.

Table 3 shows the $\mathrm{DMol}^{3}$ results for the stretch and shear that minimize energy. $E_{\text {twin }}$ is the energy difference between twin and single crystal per unit cell of the polysynthetic twin. As energy of the single crystal we took $2 \mathrm{~N}$ times the energy of a single crystal in its primitive cell. In the case $m\left(\begin{array}{lll}0 & 0 & 0\end{array}\right)$ this procedure leads to a small negative value of $E_{\text {twin }}$. To be more precise, we, instead, compared the energy of this twin with the energy of a single crystal in the supercell cell with $\mathbf{C}=2 N \mathbf{c}$.

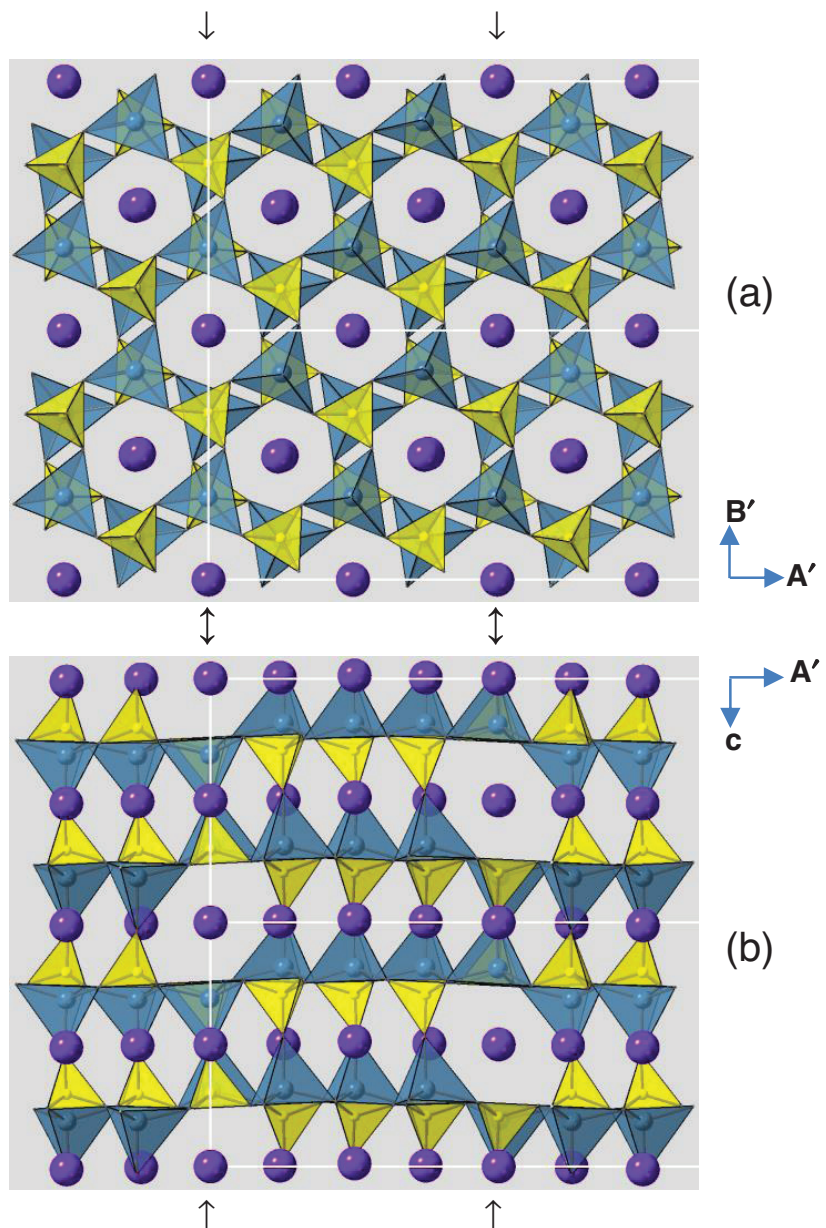

Fig. 6: DFT results for the inversion twin with boundary $(11 \overline{2} 0)$ projected || c (a) and || $\mathbf{B}^{\prime}$ (b). The arrows and double arrows indicate the boundaries; they are perpendicular to the planes of the drawings. The width of a primitive cell (marked white) is twice the distance between neighboring boundaries.

Table 3 shows that $E_{\text {twin }}$ changes only little between $N=3$ and $N=4$, so that we can assume that the value for $N=4$ is close to the value for large values of $N$.

\section{Discussion of the results and conclusions}

Considering the $N=4$ results for twin law $\overline{1}$, Table 3 shows that $E_{\text {twin }}$ is about twice as large for boundary $(11 \overline{2} 0)$ than for $(10 \overline{1} 0)$, which tells us that the energy of an inversion

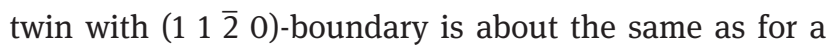

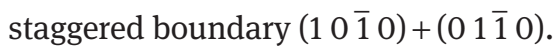

Table 3 shows that $m\left(\begin{array}{llll}0 & 0 & 0 & 1\end{array}\right)$ has by far the lowest energy. Such twins were frequently found, sometimes with boundaries traversing the whole crystal (L) and sometimes in polysynthetic form, originating from growth 
Tab. 3: The energy difference $E_{\text {twin }}$ between twin and single crystal per unit cell of the polysynthetic twin and the corresponding stretch and shear of the unit cell for polysynthetic twins with lamellae thickness $N$ times the minimum value possible.

\begin{tabular}{|c|c|c|c|c|c|c|c|c|c|c|c|c|}
\hline $\begin{array}{l}\text { Boundary plane } \\
\text { Twin law } \\
\text { Short symbol }\end{array}$ & $\begin{array}{l}\left(\begin{array}{llll}0 & 0 & 0 & 1\end{array}\right) \\
\mathrm{m} \|\left(\begin{array}{llll}0 & 0 & 1\end{array}\right] \\
\mathrm{m}\left(\begin{array}{llll}0 & 0 & 0 & 1\end{array}\right)\end{array}$ & & $\begin{array}{l}\left(\begin{array}{llll}1 & 0 & \overline{1} & 0\end{array}\right) \\
\mathrm{m} \| \\
\mathrm{m}\left(\begin{array}{llll}1 & 0 & \overline{1} & 0\end{array}\right)\end{array}$ & & $\begin{array}{l}\left(\begin{array}{llll}1 & 0 & \overline{1} & 0\end{array}\right) \\
2 \perp\left[\begin{array}{lll}0 & 0 & 1\end{array}\right] \\
2\left(\begin{array}{llll}1 & 0 & \overline{1} & 0\end{array}\right)\end{array}$ & & $\begin{array}{l}\left(\begin{array}{llll}1 & 0 & \overline{1} & 0\end{array}\right) \\
\overline{1} \\
\overline{1}\left(\begin{array}{llll}1 & 0 & \overline{1} & 0\end{array}\right)\end{array}$ & & & $\begin{array}{l}\left(\begin{array}{llll}1 & 1 & \overline{2} & 0\end{array}\right) \\
\overline{1} \\
\overline{1}\left(\begin{array}{llll}1 & 1 & \overline{2} & 0\end{array}\right)\end{array}$ & & \\
\hline \multirow{2}{*}{$\begin{array}{l}\text { Polytwin symmetry } \\
\text { Lamellae thickness } \\
N\end{array}$} & \multicolumn{2}{|r|}{ Trigonal } & \multicolumn{2}{|c|}{ Orthorhombic } & \multicolumn{2}{|c|}{ Orthorhombic } & \multicolumn{3}{|c|}{ Monoclinic } & \multicolumn{3}{|c|}{ Monoclinic } \\
\hline & $E_{\text {twin }}(\mathrm{eV})$ & $\begin{array}{r}\text { Stretch } \\
(\mathrm{pm})\end{array}$ & $E_{\text {twin }}(\mathrm{eV})$ & $\begin{array}{r}\text { Stretch } \\
(\mathrm{pm})\end{array}$ & $E_{\text {twin }}(\mathrm{eV})$ & $\begin{array}{r}\text { Stretch } \\
(\mathrm{pm})\end{array}$ & $E_{\text {twin }}(\mathrm{eV})$ & $\begin{array}{r}\text { Stretch } \\
(\mathrm{pm})\end{array}$ & $\begin{array}{r}\text { Shear } \\
(\mathrm{pm})\end{array}$ & $E_{\text {twin }}(\mathrm{eV})$ & $\begin{array}{r}\text { Stretch } \\
(\mathrm{pm})\end{array}$ & $\begin{array}{r}\text { Shear } \\
(\mathrm{pm})\end{array}$ \\
\hline 1 & 0.0008 & -0.12 & 0.36 & 31.6 & 0.31 & 28.1 & 0.06 & 8.0 & 4.1 & 0.07 & 0.5 & 2.4 \\
\hline 2 & 0.0009 & -0.15 & 0.46 & 26.3 & 0.49 & 43.0 & 0.07 & 8.1 & 2.5 & 0.15 & 8.2 & 8.2 \\
\hline 3 & 0.0009 & -0.06 & 0.57 & 48.3 & 0.54 & 47.3 & 0.08 & 8.9 & 3.1 & 0.17 & 7.9 & 3.2 \\
\hline 4 & 0.0009 & -0.13 & 0.58 & 49.4 & 0.55 & 48.9 & 0.09 & 9.2 & 3.7 & 0.17 & 8.3 & 4.5 \\
\hline
\end{tabular}

defects with lamellae often too thin to be resolved by X-ray topography (G). "L" and "G" refer to figs. 2-4 in [3], see also 5.1.3, 5.1.4 and 5.1.7 in [4]. Whereas Cr-doped crystals were considered in [3] and [4]; similar twins were found also in crystals without Cr-doping, as shown in Figs. 3a

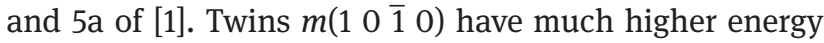

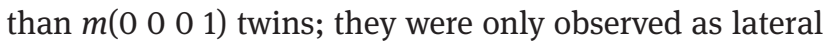
bound of ( $\left(\begin{array}{llll}0 & 0 & 0 & 1\end{array}\right)$ lamellae [3]. A similarly high energy have twins 2(10 10$)$; such twins, however, were not found. Twins $\overline{1}(10 \overline{1} 0)$ have the second lowest energy. Such twins were not found in crystals grown without $\mathrm{Cr}$ doping but frequently in Cr-doped crystals. They are marked "In" in fig. 3 of [3], see also 5.1.4 of [4].

The situation is different for $\mathrm{KLiSO}_{4}$ crystals after heating them to the high temperature phase II (stable between 708 and $949 \mathrm{~K}$ ) and cooling back to ambient temperature, as shown in [3]. Almost all $\overline{1}(10 \overline{1} 0)$ twins had disappeared. On the other hand, more $m(10 \overline{1} 0)$ twins were formed, although they have higher energy than $\overline{1}(10 \overline{1} 0)$ twins.

A similar situation exists for twins in quartz. At one bar, quartz exists as $\alpha$-quartz at ambient temperature; it reversibly transforms to $\beta$-quartz at $846 \mathrm{~K}$. Low quartz, i.e. quartz precipitated from hot hydrothermal veins was formed as $\alpha$-quartz; high quartz crystallized from molten magma as $\beta$-quartz. Energies of Brazil twins, frequent in low quartz, and of Esterel twins, the most frequent twins in high quartz, were computed using $\mathrm{DMol}^{3}$ in [19] and [20], respectively. Similarly as phase III of $\mathrm{KLiSO}_{4}$, also $\alpha$-quartz and $\beta$-quartz have a hexagonal lattice. Whereas the point group $6 / \mathrm{mmm}$ of the hexagonal lattice has order 24 , the point groups 6 of phase III and 32 of $\alpha$-quartz have order 6 (tetartohedry). Table 4 compares the various twin laws and boundary planes considered with DMol${ }^{3}$.

Brazil twins with boundary plane parallel to the major rhombohedron $\mathrm{r}$ occur very frequently in low quartz, especially in amethyst, which contains $\mathrm{Fe}_{2} \mathrm{O}_{3}$ in the \%o-range [21]. This reminds of $\mathrm{KLiSO}_{4}$, where, again, the inversion twin was promoted by doping in the \%o-range. Brazil twins with boundary plane parallel to the minor rhombohedron $\mathrm{z}$ are rare [21], as had to be expected from the much higher energy. Esterel twins in low quartz (usually referred to as Reichenstein-Grieserntal twins) are very rare in accordance with their large value $E_{\mathrm{twin}} / A=1.49 \mathrm{eV} / \mathrm{nm}^{2}$.

Tab. 4: $\mathrm{DMol}^{3}$ results for twins in $\mathrm{KLiSO}_{4}$ and quartz listed in the order of increasing energy difference between twin and single crystal $E_{\text {twin }}$ divided by boundary plane area $A$.

\begin{tabular}{|c|c|c|c|c|c|}
\hline Crystal & Twin law & Boundary plane & Space group of polysynthetic twin & Lattice index $\Sigma$ & $E_{\mathrm{twin}} / A\left(\mathrm{eV} / \mathrm{nm}^{2}\right)$ \\
\hline $\mathrm{KLiSO}_{4}$, phase III & $m \|[001]$ & $\left(\begin{array}{llll}0 & 0 & 0 & 1\end{array}\right)$ & P31c (\#159) & 1 & 0.002 \\
\hline$\alpha$-quartz & $\overline{1}=$ Brazil & $\left(\begin{array}{llll}1 & 0 & \overline{1} & 1\end{array}\right)=r$ & $C 2 / c(\# 15)$ & 1 & 0.041 \\
\hline $\mathrm{KLiSO}_{4}$, phase III & $\overline{1}$ & $\left(\begin{array}{llll}1 & 0 & \overline{1} & 0\end{array}\right)$ & $P 2_{1} / c(\# 14)$ & 1 & 0.093 \\
\hline$\alpha$-quartz & $\overline{1}=$ Brazil & $\left(\begin{array}{llll}0 & 1 & \overline{1} & 1\end{array}\right)=z$ & C2/c (\#15) & 1 & 0.389 \\
\hline $\mathrm{KLiSO}_{4}$, phase III & $2 \perp[001]$ & $\left(\begin{array}{llll}1 & 0 & \overline{1} & 0\end{array}\right)$ & $P 2_{1} 2_{1} 2$ (\#18) & 1 & 0.605 \\
\hline $\mathrm{KLiSO}_{4}$, phase III & $m \|[001]$ & $\left(\begin{array}{llll}1 & 0 & \overline{1} & 0\end{array}\right)$ & Pca2 $_{1}(\# 29)$ & 1 & 0.638 \\
\hline$\beta$-quartz & Esterel (reflection) & $(0 \overline{1} 111)$ & Fdd2 (\#43) & 3 & 1.02 \\
\hline$\alpha$-quartz & "Esterel” (reflection) & $(0 \overline{1} 11)$ & Fdd2 (\#43) & 3 & 1.49 \\
\hline
\end{tabular}

A lattice index $\Sigma=1$ corresponds to merohedric twinning, whereas the principal axes of the two individuals of an Esterel twin are not parallel. The row " $\beta$-quartz" shows the results of our calculations for the $\beta$-quartz structure, stable in the temperature range $846-1143 \mathrm{~K}$ at atmospheric pressure. 
Looking at high quartz, which transformed from the $\beta$-quartz of solidified magma to $\alpha$-quartz at ambient temperature, Esterel twins are the most common twins although their energy is high, whereas Brazil twins with their low energy are not known [21]. The frequency of twins in high quartz depends more on the availability of twin nuclei than on the energy of the twin boundary.

Also the frequency of twins in $\mathrm{KLiSO}_{4}$ after heating completely to phase II and cooling back to phase III shows little correlation with twinning energy: the low energy twins $\overline{1}(10 \overline{1} 0)$ have disappeared whereas the area of $m(10 \overline{1} 0)$ twin boundaries has increased despite their much higher energy [3].

Acknowledgments: The authors are indebted to Prof. H. Klapper for proposing this investigation, for answering their questions and for a copy of Ref. [4].

\section{References}

[1] H. Klapper, Th. Hahn, S. J. Chung, Optical, pyroelectric and X-ray topographic studies of twin domains and twin boundaries in $\mathrm{KLiSO}_{4}$. Acta Cryst. 1987, B43, 147, with Erratum on page 406 of the same volume.

[2] H. Klapper, H.-D. Jennissen, Chr. Scherf, Th. Hahn, X-ray topographic and polarisation-optical studies of the low-temperature phase transitions and domain structures of $\mathrm{KLiSO}_{4}$. Ferroelectrics 2008, 376, 25.

[3] Chr. Scherf, N. R. Ivanov, S. J. Chung, Th. Hahn, H. Klapper, High - temperature phase transitions and domain structures of $\mathrm{KLiSO}_{4}$ : studied by polarisation-optics, $\mathrm{X}$-ray topography and liquid-crystal surface decoration. Z. Kristallogr. 2017, 232, 415.

[4] Chr. Scherf, Strukturelle Phasenübergänge und Zwillingsdomänen des Kaliumlithium-sulfats und verwandter Sulfate. Doctoral Thesis, RWTH Aachen, 2000. 286 pages, in German. Shaker Verlag, Aachen 2000, ISBN 3-8265-8258-6. http://publications. rwth-aachen.de/record/96444/files/Scherf_Christian.pdf.

[5] H. Schulz, U. Zucker, R. Frech, Crystal structure of $\mathrm{KLiSO}_{4}$ as a function of temperature. Acta Cryst. 1985, B41, 21.

[6] J. F. Nye, Physical properties of crystals. Clarendon Press, Oxford 1985.
[7] B. Delley, From molecules to solids with the $\mathrm{DMol}^{3}$ approach. J. Chem. Phys. 2000, 113, 7756.

[8] International Tables for Crystallography, Vol. A. Space-group symmetry, (Ed. M. I. Aroyo) 6th edition, International Union of Crystallography. John Wiley \& Sons, Ltd, Chichester, UK, 2016.

[9] J. P. Perdew, Y. Wang, Accurate and simple analytic representation of the electron-gas correlation energy. Phys. Rev. 1992, B45, 13244 .

[10] J. P. Perdew, K. Burke, M. Ernzerhof, Generalized gradient approximation made simple. Phys. Rev. Lett. 1996, 77, 3865.

[11] J. P. Perdew, A. Ruzsinszky, G. I. Csonka, O. A. Vydrov, G. E. Scuseria, L. A. Constantin, X. Zhou, K. Burke, Restoring the density-gradient expansion for exchange in solids and surfaces. Phys. Rev. Lett. 2008, 100, 136406, with Erratum in Phys. Rev. Lett. 2009, 102, 039902.

[12] J. Sun, A. Ruzsinszky, J. P. Perdew, Strongly constrained and appropriately normed semilocal density functional. Phys. Rev. Lett. 2015, 115, 036402.

[13] A. Jain, S. P. Ong, G. Hautier, W. Chen, W. D. Richards, S. Dacek, S. Cholia, D. Gunter, D. Skinner, G. Ceder, K. A. Persson, The materials project: a materials genome approach to accelerating materials innovation. APL Mater. 2013, 1, 011002.

[14] M. A. Pimenta, Y. Luspin, G. Hauret, Brillouin light scattering in LiKSO ${ }_{4}$ between 20 and $80^{\circ} \mathrm{C}$. Solid State Commun. 1986, $59,481$.

[15] H. Kabelka, G. Kuchler, Elastic stiffness constants and elastic relaxation around the first low temperature phase transition in LiKSO $_{4}$. Ferroelectrics 1988, 88, 93.

[16] L. Godfrey, J. Philip, Ultrasonic measurement of the elastic constants of LiKSO ${ }_{4}$ between 300 and 370 K. Solid State Commun. 1996, 97, 635.

[17] F. Willis, R. G. Leisure, T. Kanashiro, Temperature dependence of the elastic constants of $\mathrm{LiKSO}_{4}$ through a first-order structural phase transition. Phys. Rev. 1996, B54, 9077.

[18] Th. Hahn, H. Klapper, Twinning of crystals. Chapter 3.3, in International Tables for Crystallography, Vol. D. Physical Properties of Crystals, (Ed. A. Authier) Second Edition, International Union of Crystallography. John Wiley \& Sons, Ltd, Chichester, UK, 2014.

[19] H. Grimmer, B. Delley, Density functional calculations of polysynthetic Brazil twinning in $\alpha$-quartz. Acta Cryst. 2012, A68, 359, with Erratum in Acta Cryst. 2014, A70, 682.

[20] B. Delley, H. Grimmer, Density-functional calculations of Esterel twinning in quartz. Phys. Rev. 2007, B76, 224106.

[21] C. Frondel, The system of mineralogy, Vol. III. Silica Minerals, 7th edition. John Wiley \& Sons, Inc., New York and London, 1962. 